\title{
Three-loop vertex integrals at symmetric point
}

\author{
Andrey Pikelner \\ Bogoliubov Laboratory of Theoretical Physics, Joint Institute for Nuclear Research, \\ Dubna 141980, Russia \\ Theory department, Budker Institute of Nuclear Physics, \\ Novosibirsk 630090, Russia \\ E-mail: pikelner@theor.jinr.ru
}

\begin{abstract}
This paper provides details of the massless three-loop three-point integrals calculation at the symmetric point. Our work aimed to extend known two-loop results for such integrals to the three-loop level. Obtained results can find their application in regularization-invariant symmetric point momentum-subtraction (RI/SMOM) scheme QCD calculations of renormalization group functions and various composite operator matrix elements. To calculate integrals, we solve differential equations for auxiliary integrals by transforming the system to the $\varepsilon$-form. Calculated integrals are expressed through the basis of functions with uniform transcendental weight. We provide expansion up to the transcendental weight six for the basis functions in terms of harmonic polylogarithms with six-root of unity argument.
\end{abstract}

KEYwords: Lattice QCD, Renormalization Group

ArXiv EPrint: 2104.06958 


\section{Contents}

1 Introduction 1

2 Notations for integrals topologies 3

3 Differential equations for auxiliary integrals 4

4 Fixing boundary conditions $\quad 6$

5 Basis of integrals with uniform transcendentality weight 9

6 Results and conclusion $\quad 10$

\section{Introduction}

Many physically significant quantities in QCD can be extracted from the three-point Green functions. There is remarkable progress $[1-4]$ in calculating QCD renormalization group functions in the minimal subtraction $(\overline{\mathrm{MS}})$ renormalization scheme [5]. We can choose exceptional momenta routing(one of the external momenta set to zero) and restrict ourselves to considering only the divergent parts of diagrams for these calculations. Due to the unphysical nature of the minimal subtraction scheme, a group of momentum subtraction schemes(MOM) [6] is widely used in calculations requiring finite parts of the three-point functions. Such physical schemes are crucial for the Lattice calculations, where one has access to vertex functions directly. Calculation of various vertices in regularization invariant momentum subtraction $(\mathrm{RI} / \mathrm{MOM})$ schemes provides a connection between the lattice calculations and the MS scheme results. By choosing exceptional momenta routing, putting one of the external momenta to zero, we define RI/MOM scheme. With symmetric point kinematics configuration with all squares of external momenta equal, we define RI/SMOM scheme.

The main difficulty in the RI/MOM and RI/SMOM scheme calculation is the necessity to know finite parts of vertex functions in the chosen kinematics. If we require only finite parts of the propagator type integrals for the exceptional momenta routing, then for the vertices in the RI/SMOM case, we need to know three-point integrals at the symmetric point. Calculation of the symmetric point integrals is the main difficulty of the RI/SMOM scheme. The present paper focuses on how to solve the problem at the three-loop level.

There are many results based on the calculation of the two-loop three-point functions at the symmetric point. Namely three-loop RI/SMOM beta-functions [7], two-loop correction to the relation between RI/SMOM and MS quark mass [8, 9], and renormalization of Wilson operator matrix elements [7-11]. We recently extended some of these calculations to the three-loop level analytically [12, 13], using results of the present paper. Independently, 

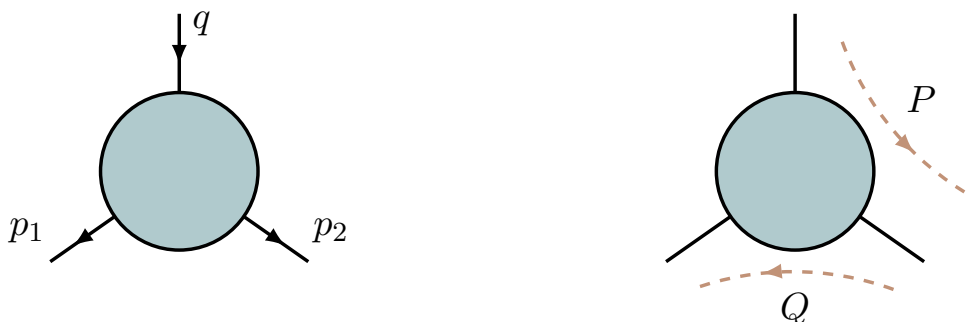

Figure 1. External momenta assignment for auxiliary and symmetric point integrals(left) and for the large momentum expansion procedure(right).

numerical results for renormalization of different operators matrix elements appeared in the series of papers $[14,15]$.

Integrals of our interest are given on the left of the figure 1 and condition $p_{1}^{2}=p_{2}^{2}=$ $q^{2}=-\mu^{2}$ defines the symmetric subtraction point. From now on, we put $\mu^{2}=1$ to simplify the calculation, but one can easily reconstruct integrals values for an arbitrary symmetric point $\left(\mu^{2} \neq 1\right)$ from dimensional considerations and known powers of propagators.

Before providing details of the three-loop calculation, we need to review techniques used at two loops. The first two-loop calculation at the symmetric point [16] used a large momentum expansion procedure. This technique does not rely on any symmetric point integrals knowledge and requires only results for massless propagator integrals. In subsequent two-loop calculations [7-11], authors adopted Integration-By-Parts(IBP) [17, 18] reduction to the minimal set of two-loop master integrals known for a long time, even in general kinematics not restricted to the symmetric point [19-21]. This way, the problem of the master integrals calculation had not appeared explicitly in those works.

On the other hand, a very restricted subset of the three-loop master integrals needed for analytical calculations at the symmetric point $[12,13]$ is known [22].

Our starting point for three-loop master integrals calculation is a paper [23], where linear reducibility of the two-loop three-point integrals with off-shell legs is demonstrated after an appropriate variable change. Linear reducibility of three-loop integrals with offshell kinematics was indicated in the E.Panzer Ph.D. thesis [24]. Since symmetric point integrals are a particular case of considered integrals, we expect them to be expressed in terms of generalized polylogarithms(GPL) as a direct consequence of the linear reducibility. The same property will have auxiliary integrals with $p_{1}^{2}=p_{2}^{2}$, but $q^{2}$ arbitrary, and we will be able to reduce differential equations (DE) system for such integrals to the so-called $\varepsilon$-form [25].

As the primary calculation method, we solve differential equations system for auxiliary integrals. Due to performance issues with the most complicated integrals, we use direct integration in terms of GPLs for partial checks only. Also, the method of direct integration requires construction of a new set of master integrals without sub-divergences, which is possible to achieve by shifting space-time dimension and increasing some propagators powers [26]. Conversion between the set of finite integrals and the original basis is an additional complication of the method. However, an alternative based on the DE system solution is 
also highly nontrivial. We need to consider more complicated integrals depending on at least one dimensionless variable and provide boundary conditions(BC) sufficient to fix all needed integration constants in the DE solution.

The paper organized as follows: we define auxiliary topologies containing all integrals of our interest in section 2, we describe the solution of differential equations for auxiliary integrals in section 3, we describe our approach to fixing boundary conditions with a detailed two-loop example in section 4 , construction of the uniform transcendentality weight basis of integrals can be found in section 5 , and in section 6 we provide the list of calculated master integrals. Actual results for the integrals are in the supplementary materials to the paper.

\section{Notations for integrals topologies}

To reduce many integrals in the course of calculations $[12,13]$, we apply IBP reduction to the small set of master integrals considered in the present paper. To perform the reduction efficiently, we use the Laporta algorithm [27] implemented in the package Reduze2 [28]. We define auxiliary topologies containing the complete set of propagators to express all the appearing scalar products for the algorithm's work. To uniquely identify an integral inside topology, we use a vector of integer numbers corresponding to propagators' powers.

For a vertex integral at the symmetric point, we assign external momenta according to the left part of the figure 1 and set $p_{1}^{2}=p_{2}^{2}=q^{2}=-1$. This definition makes all integrals real, and self-energy integrals are in one-to-one correspondence with integrals from the MINCER package $[29,30]$. Later, this property will be helpful for the boundary conditions fixing procedure described in section 4 .

At the one-loop level, we have a single topology $A_{1}$ with three propagators

$$
I_{A_{1}}\left[n_{1}, n_{2}, n_{3}\right]=e^{\varepsilon \gamma_{E}} \int \frac{d^{D} k_{1}}{i \pi^{D / 2}} \frac{1}{\left(\left(k_{1}+p_{1}\right)^{2}\right)^{n_{1}}\left(\left(k_{1}-p_{2}\right)^{2}\right)^{n_{2}}\left(k_{1}^{2}\right)^{n_{3}}} .
$$

At the two-loop level, we have two seven-propagator topologies, $A_{2}$ and $B_{2}$

$$
I_{X_{2}}\left[n_{1}, \ldots, n_{7}\right]=e^{2 \varepsilon \gamma_{E}} \int \frac{d^{D} k_{1}}{i \pi^{D / 2}} \frac{d^{D} k_{2}}{i \pi^{D / 2}} \frac{1}{\left(P_{1}^{2}\right)^{n_{1}} \ldots\left(P_{7}^{2}\right)^{n_{7}}},
$$

where $X=\{A, B\}$ and propagators' momenta are defined explicitly in table 1 .

At three-loops, we have three topologies, $A_{3}, B_{3}$, and $C_{3}$, each with 12 propagators

$$
I_{X_{3}}\left[n_{1}, \ldots, n_{12}\right]=e^{3 \varepsilon \gamma_{E}} \int \frac{d^{D} k_{1}}{i \pi^{D / 2}} \frac{d^{D} k_{2}}{i \pi^{D / 2}} \frac{d^{D} k_{3}}{i \pi^{D / 2}} \frac{1}{\left(P_{1}^{2}\right)^{n_{1}} \ldots\left(P_{12}^{2}\right)^{n_{12}}} .
$$

Table 2 provides explicit expressions for momenta $P_{i}$ for each of the three-loop topologies with $X=\{A, B, C\}$.

The number of unique master integrals after IBP reduction are given in table 3 . We present all master integrals graphs in tables 4,5 and 6 . 


\begin{tabular}{|rrr|}
\hline & $A_{2}$ & $B_{2}$ \\
\hline$P_{1}$ & $k_{1}+p_{1}$ & $k_{1}+p_{1}$ \\
$P_{2}$ & $k_{2}+p_{1}$ & $k_{2}+p_{1}$ \\
$P_{3}$ & $k_{1}-p_{2}$ & $k_{1}-p_{2}$ \\
$P_{4}$ & $k_{2}-p_{2}$ & $k_{1}-k_{2}-p_{2}$ \\
$P_{5}$ & $k_{1}-k_{2}$ & $k_{1}-k_{2}$ \\
$P_{6}$ & $k_{1}$ & $k_{2}$ \\
$P_{7}$ & $k_{2}$ & $k_{1}$ \\
\hline
\end{tabular}

Table 1. Two-loop auxiliary topologies.

\begin{tabular}{|rrrr|}
\hline & $A_{3}$ & $B_{3}$ & $C_{3}$ \\
\hline$P_{1}$ & $k_{1}$ & $k_{1}$ & $k_{1}$ \\
$P_{2}$ & $k_{2}$ & $k_{2}$ & $k_{2}$ \\
$P_{3}$ & $k_{3}$ & $k_{3}$ & $k_{3}$ \\
$P_{4}$ & $k_{1}-k_{2}$ & $k_{1}-k_{2}$ & $k_{1}-k_{2}$ \\
$P_{5}$ & $k_{1}-k_{3}$ & $k_{1}-k_{3}$ & $k_{1}-k_{3}$ \\
$P_{6}$ & $k_{2}-k_{3}$ & $k_{1}-k_{2}-k_{3}$ & $k_{2}-k_{3}$ \\
$P_{7}$ & $k_{1}-p_{1}$ & $k_{1}-p_{1}$ & $k_{1}-k_{3}-p_{2}$ \\
$P_{8}$ & $k_{1}-p_{1}-p_{2}$ & $k_{1}-p_{1}-p_{2}$ & $k_{1}-p_{1}-p_{2}$ \\
$P_{9}$ & $k_{2}-p_{1}$ & $k_{2}-p_{1}$ & $k_{2}-p_{1}$ \\
$P_{10}$ & $k_{2}-p_{1}-p_{2}$ & $k_{2}-p_{1}-p_{2}$ & $k_{1}-k_{2}-p_{2}$ \\
$P_{11}$ & $k_{3}-p_{1}$ & $k_{3}-p_{1}$ & $k_{3}-p_{1}$ \\
$P_{12}$ & $k_{3}-p_{1}-p_{2}$ & $k_{3}-p_{1}-p_{2}$ & $k_{3}-p_{1}-p_{2}$ \\
\hline
\end{tabular}

Table 2. Three-loop auxiliary topologies.

\begin{tabular}{|c||c|c||c|c|c|}
\hline$A_{1}$ & $A_{2}$ & $B_{2}$ & $A_{3}$ & $B_{3}$ & $C_{3}$ \\
\hline 2 & 7 & 1 & 38 & 12 & 1 \\
\hline
\end{tabular}

Table 3. Distribution of the number of integrals over topologies.

\section{Differential equations for auxiliary integrals}

As stated in the introduction, our main calculation method is a solution of the DE system for specially constructed auxiliary integrals. We consider integrals similar to original threepoint ones, but now with $q^{2}$ not fixed, and introduce scaleless variable $x$ with $q^{2}=x p_{1}^{2}$. At first glance, this makes us calculate more complex integrals than the initial set. However, the advantage is that now we have access to the DE system, connecting point $x=1$ with any other point, where boundary conditions can be constructed easily. In our calculation, we consider the limit $x \rightarrow 0$ for fixing boundary conditions and the limit $x \rightarrow \infty$ for checks.

We analyze the same topologies as before, but now with $q^{2}$ not fixed for auxiliary integrals. After performing IBP reduction and identification of master integrals for each of topologies, we have the following number of master integrals: 3 for $A_{1}, 12$ for $A_{2}, 11$ for $B_{2}, 88$ for $A_{3}$ and 91 for $B_{3}$. Since for topology $C_{3}$ at the symmetric point, we have only 
one integral, which factorizes into lower-loop integrals from topologies $A_{1}$ and $B_{2}$, we do not consider it here.

To differentiate master integrals in variable $x$ and reduce the result back to master integrals, we make use of the package Reduze 2 [28]. To convert the obtained DE system to the $\varepsilon$-form with first apply a variable change $x=-\frac{(z-1)^{2}}{z}$. In a new variable $z$, singular points of the DE system correspond to the following set of singular points in an old variable $x$ :

$$
z \rightarrow e^{ \pm \frac{i \pi}{3}}: x \rightarrow 1, \quad z \rightarrow 1: x \rightarrow 0, \quad z \rightarrow 0: x \rightarrow \infty, \quad z \rightarrow-1: x \rightarrow 4
$$

The point $z=e^{ \pm \frac{i \pi}{3}}$ is a solution we are interested in, limit $z=1$ we use in section 4 as boundary conditions to fix integration constants in the DE solution. The solution in the point $z=0$ is used to derive known results for the there-loop massless form-factor type integrals [31-33], which is necessary for checking obtained results. We do not consider the point $z=-1$ in the present paper.

After the variable change, the system of differential equations for the vector of integrals $\vec{f}(z, \varepsilon)$ for each of the considered topologies has the following form

$$
\partial_{z} \vec{f}(z, \varepsilon)=M(z, \varepsilon) \vec{f}(z, \varepsilon) .
$$

With matrix $M$ having only a limited set of $\varepsilon$-independent singularities of the finite order in the set of points $S=\left\{0,1,-1, \lambda, \lambda^{*}\right\}$, where $\lambda=e^{\frac{i \pi}{3}}$ and $\lambda^{*}$ is its complex conjugate.

$$
M(z, \varepsilon)=\sum_{z_{j} \in S} \sum_{k \geq 0} \frac{M_{k}^{\left(z_{j}\right)}(\varepsilon)}{\left(z-z_{j}\right)^{k+1}}+\sum_{k \geq 0} z^{k} M_{k}(\varepsilon)
$$

This form of the DE system is ideally suited for further conversion to the canonical form [25]. Only one complication is the appearance of sixth-roots of unity residues at the three-loop order. For one and two-loop integrals, the DE systems are singular only in $z=\{0,1,-1\}$.

Following the strategy from [25] and with algorithm [34] implemented in the publicly available package epsilon [35], we convert the original DE system to another DE system for a new basis of integrals $\vec{g}(z, \varepsilon)=T^{-1}(z, \varepsilon) \vec{f}(z, \varepsilon)$

$$
\partial_{z} \vec{g}(z, \varepsilon)=\varepsilon\left[\frac{A_{0}}{z}+\frac{A_{1}}{z-1}+\frac{A_{-1}}{z+1}+\frac{A_{\lambda}}{z-\lambda}+\frac{A_{\lambda^{*}}}{z-\lambda^{*}}\right] \vec{g}(z, \varepsilon) .
$$

Obtained DE system matrix has only Fuchsian singularities, and $\varepsilon$ dependence completely factorizes. Applying epsilon for reduction of the original system to the form (3.4), we perform all the steps of the algorithm [34] except the last one. Due to the performance issues, we were forced to find a constant matrix transforming the system to the form with $\varepsilon$ factorized manually. Another reason to make the last step manually is that the found transformation matrix is not unique, as not unique the DE system matrix in $\varepsilon$-form. This freedom in the transformation matrix and related freedom in the $\varepsilon$-form matrix we use in section 5, where we construct a new basis of integrals with uniform transcendentality (UT) weight. 
The differential equations system (3.4) has an excellent property that, after expanding all the master integrals in $\varepsilon$, the differential equations for the series coefficients $\vec{g}_{i}(z)$ with $\vec{g}(z, \varepsilon)=\sum \vec{g}_{i}(z) \varepsilon^{i}$ decouple entirely, and the solution for the particular coefficient has the form:

$$
\vec{g}_{i}(z)=\int_{0}^{z} d y\left[\frac{A_{0}}{y}+\frac{A_{1}}{y-1}+\frac{A_{-1}}{y+1}+\frac{A_{\lambda}}{y-\lambda}+\frac{A_{\lambda^{*}}}{y-\lambda^{*}}\right] \vec{g}_{i-1}(y)+\vec{r}_{i}
$$

To perform integration, we utilize GPLs [36] integration rules, and starting with some order $n$ of $\varepsilon$-expansion where all $\vec{g}_{n} \equiv 0$ we proceed by induction. At the next step we associate integration constants with zero weight GPLs, since $G(; z) \equiv 1$. Singularities of the system (3.4) define the alphabet of GPLs. In the examined case, we have a subset of the full six-root-of-unity alphabet considered in [37], and for lower loop orders, the alphabet of HPLs containing only $\{0,1,-1\}$ is enough. GPLs are directly integrated with

$$
G\left(a_{1}, \ldots, a_{n} ; z\right)=\int_{0}^{z} \frac{d t}{t-a_{1}} G\left(a_{2}, \ldots, a_{n} ; t\right) .
$$

Each order of $\varepsilon$-expansion of the constructed solution is built from the GPLs of the uniform transcendental weight multiplied by some unknown constants. In the general case, actual values of integration constants $\vec{r}_{i}$ can spoil the uniform transcendentality(UT) structure of the solution, and we address the question of restoring UT expansion in section 5. Our next step is to fix integration constants $\vec{r}_{i}$ by providing appropriate boundary conditions.

\section{Fixing boundary conditions}

To fix integration constants in the solution of DE from the previous section, we consider its behavior near the point $z=1$. Since the point $z=1$ is a singular point of the DE system (3.4), we can not take the limit directly due to the logarithmic singularities $\log (1-z)$ appearing in the solution. Thanks to the Fuchsian form of the DE system (3.4), the leading order term of expansion in a small variable $\bar{z}=1-z$ can be constructed directly from the matrix residue $A_{1}[38]:^{1}$

$$
\lim _{\bar{z} \rightarrow 0} \vec{g}(\varepsilon, z)=\bar{z}^{\varepsilon A_{1}} \vec{c}(\varepsilon)+\mathcal{O}(\bar{z}) .
$$

This leading term of the solution is exact in $\varepsilon$, and vector of unknown $\varepsilon$-dependent functions $\vec{c}(\varepsilon)$ has the same size as the vector of master integrals $\vec{g}(\varepsilon, z)$. Knowledge of the vector of functions $\vec{c}(\varepsilon)$ up to sufficiently high order in $\varepsilon$ is enough to fix all needed integration constants. The series solution in the form (4.1) allows us to separate contribution from different subgraphs by considering terms with different non-integer powers of $\bar{z}$. It is handy to extract contribution from the hard subgraph, accessible from the naive Taylor expansion of the integrand under the integral sign. However, in the considered case, naive expansion is not enough to fix all required integration constants, and we need to consider asymptotic expansion, including all relevant subgraphs. To calculate the vector of functions $\vec{c}(\varepsilon)$, we develop a highly automated setup based on the package EXP [40, 41] and a

\footnotetext{
${ }^{1}$ Higher orders of expansion in $\bar{z}$ can be constructed recursively, as shown in [39].
} 
version of the MINCER $[29,30]$ package keeping all intermediate $\varepsilon$-dependent expressions in unexpanded form.

Momenta routing used for the asymptotic expansion coefficients calculation is given in figure 1(right). With this routing, our kinematics constraints are as follows $P^{2}=x Q^{2}$ and $P \cdot Q=\frac{x}{2} Q^{2}$, and results of the expansion in the limit $x \rightarrow 0$ we can obtain from the large momentum procedure(LMP) considering $Q$ large. For more details on the LMP, see [42] and references therein. Expansion of the arbitrary three-point vertex integral with external momenta $P, Q$ in large momentum $Q$ has the form:

$$
J(P, Q) \sim \sum_{S}\left(\frac{P^{2}}{Q^{2}}\right)^{\varepsilon S} \sum_{i, j} a_{i, j}^{S}\left(\frac{P \cdot Q}{Q^{2}}\right)^{i}\left(\frac{P^{2}}{Q^{2}}\right)^{j}
$$

To fix coefficients $a_{i, j}^{S}$ of the expansion, we use setup based on the package EXP. First, using EXP, we identify all needed subgraphs to expand in large external momenta, then we expand to sufficiently high order in the large momentum $Q$, further we perform tensor reduction to separate powers of the scalar products $Q^{2}$ and $P \cdot Q$ from loop integrals dependent on a single external momenta $Q$. The remaining two-point integrals are calculated with MINCER, which is fast enough to provide us with expansion terms to high order in variable $\bar{z}$. We determine coefficient $a_{i, j}$ as a linear combination of massless propagator-type master integrals, these integrals we can expand later up to the required order in $\varepsilon$.

After conversion of obtained expansions back with $T^{-1}$, we truncate the series to get the leading term for $\vec{g}$ and fix $\vec{c}(\varepsilon)$ from eq. (4.1). Contributions of different subgraphs from different integrals to the same set of functions $\vec{c}$ provide us with a strong check on the validity of the procedure.

Now expanding both solution of the system (3.4) in $z \rightarrow 1$ and leading order solution (4.1) with $\vec{c}(\varepsilon)$ substituted, we can fix all integration constants up to required order in $\varepsilon$ simply expanding MINCER master integrals deep enough in $\varepsilon$.

As a real example, we consider the DE system for non-trivial two-loop integrals

$$
J_{1}=I_{0,1,0,0,1,1,0}^{A 2}, \quad J_{2}=I_{0,1,1,0,1,0,0}^{A 2}, \quad J_{3}=I_{0,1,1,0,1,1,0}^{A 2}, \quad J_{4}=I_{0,2,1,0,1,1,0}^{A 2} .
$$

Integrals $\vec{J}$ are transformed to the canonical basis integrals $\vec{j}=T^{-1} \vec{J}$ with the matrix

$$
T=\left(\begin{array}{cccc}
\frac{-2 \varepsilon^{3}}{(1-2 \varepsilon)(2-3 \varepsilon)(1-3 \varepsilon)} & 0 & 0 & 0 \\
0 & \frac{-2 \varepsilon^{3}(1-z)^{2}}{(1-2 \varepsilon)(2-3 \varepsilon)(1-3 \varepsilon) z} & 0 & 0 \\
0 & 0 & \frac{2 \varepsilon^{2}}{(1-2 \varepsilon)(1-3 \varepsilon)} & \frac{\varepsilon^{2} z}{(1-2 \varepsilon)(1-3 \varepsilon)(1+z)} \\
0 & 0 & 0 & \frac{-\varepsilon z}{(1-z)(1+z)}
\end{array}\right) .
$$

DE system for integrals $\vec{j}(z)$ now is in the $\varepsilon$-form

$$
\partial_{z} \vec{j}(z)=\varepsilon\left(\frac{A_{0}}{z}+\frac{A_{1}}{z-1}+\frac{A_{-1}}{z+1}\right) \vec{j}(z)
$$

with numeric matrices

$$
A_{0}=\left(\begin{array}{cccc}
0 & 0 & 0 & 0 \\
0 & 2 & 0 & 0 \\
0 & -1 & 1 & 0 \\
2 & 4 & -2 & -1
\end{array}\right), \quad A_{1}=\left(\begin{array}{cccc}
0 & 0 & 0 & 0 \\
0 & -4 & 0 & 0 \\
-1 & 0 & -1 & 0 \\
0 & 0 & 0 & -1
\end{array}\right), \quad A_{-1}=\left(\begin{array}{cccc}
0 & 0 & 0 & 0 \\
0 & 0 & 0 & 0 \\
0 & 0 & 0 & -\frac{1}{2} \\
0 & 0 & 0 & 2
\end{array}\right)
$$


Due to the Fuchsian form of the DE system, the leading order solution in the limit $z \rightarrow 1$ is determined by the matrix exponent:

$$
\lim _{\bar{z} \rightarrow 0} \vec{j}(\varepsilon, z)=\bar{z}^{\varepsilon A_{1}} \vec{c}(\varepsilon)+\mathcal{O}(\bar{z}) .
$$

With $\bar{z}=1-z$ it reads when we keep leading terms in $\bar{z}$ only

$$
j_{1}=c_{1}, \quad j_{2}=\bar{z}^{-4 \varepsilon} c_{2}, \quad j_{3}=-c_{1}+\bar{z}^{-\varepsilon}\left(c_{1}+c_{3}\right), \quad j_{4}=\bar{z}^{-\varepsilon} c_{4} .
$$

Here all $c_{i}$ are functions of $\varepsilon$, and we see that some of the functions $c_{i}$ can be fixed independently from different integrals expansions stemming as an additional cross check. In provided example $c_{1}$ can be fixed both from the $j_{1}$ and $j_{3}$. With EXP we obtain the following expansions for original integrals:

$$
J_{1}=T_{1}, \quad J_{2}=\left(-\bar{z}^{2}\right)^{1-2 \varepsilon} T_{1}+\mathcal{O}\left(\bar{z}^{3}\right), \quad J_{3}=\frac{2-3 \varepsilon}{\varepsilon} T_{1}+\mathcal{O}(\bar{z}), \quad J_{4}=\mathcal{O}(\bar{z})
$$

Where $T_{1}$ is the two-loop massless sunset integral with $Q^{2}=-1$. Above we provide only orders needed to fix all $c_{i}(\varepsilon)$ entering (4.8). After multiplication with $T^{-1}$ we find the required functions:

$$
c_{1}=-\frac{(1-2 \varepsilon)(2-3 \varepsilon)(1-3 \varepsilon)}{2 \varepsilon^{3}} T_{1}, \quad c_{2}=(-1)^{1-2 \varepsilon} c_{1}, \quad c_{3}=-c_{1}, \quad c_{4}=0
$$

After fixing the required integration constants, the obtained solution can be checked by considering the limit $z \rightarrow 0$, corresponding to infinitely large $\left(q^{2} \rightarrow \infty\right)$ external momentum squared. The hard subgraph's contribution in this limit allows us to extract results for the massless form-factor integrals and compare them with expressions known up to the threeloop order [32, 33].

As before due to the Fuchsian form of the DE system, we can follow procedure described in [39] and construct general form of the expansion in $z \rightarrow 0$

$$
g(z, \varepsilon)=\sum_{i} z^{a_{i} \varepsilon} \sum_{j=0} c_{j}(\varepsilon) z^{j}
$$

Expanding integrals $\vec{g}(z)$ in $\varepsilon$, we can find several first terms of a Laurent expansion of $c_{j}(\varepsilon)$ around $\varepsilon=0$. With the help of the matrix $T$, we can construct expansion for the original basis of integrals $\vec{f}$, which we utilize to extract terms with specific noninteger powers of $z$. We can extract the value $I_{\mathrm{FF}}$ for the massless form-factor integral with $L$-loops and $E$ internal edges from the hard subgraph contribution of the form:

$$
J_{\text {hard }}=(-z)^{E-2 L+L \varepsilon}\left(I_{\mathrm{FF}}+\mathcal{O}(z)\right)
$$

From the present paper results, we found agreement with the results of the paper [33] for all master integrals up to the transcendental weight six. 


\section{Basis of integrals with uniform transcendentality weight}

The main problem with the already obtained solution is that its expansion coefficients lack the UT property. For practical applications like [12, 13], we need to expand some of the integrals to high order in $\varepsilon$ corresponding to the transcendental weight seven. Only part of the integrals with GPLs up to the transcendental weight six enters the final result, and all GPLs with transcendental weight seven cancel in the sum. At intermediate steps, manipulations with a weight seven GPLs are highly complicated, since reduction rules are available up to the weight six only [37].

Possible solution to the problem is a new basis of UT weight functions, which need to be known up to the transcendentality weight six to express results containing GPLs up to the weight six. Explicitly we want to find a basis of pure functions $\vec{U}=T_{\mathrm{UT}}^{-1} \vec{f}$, with $\varepsilon$-expansion in the form $\vec{U}=\sum_{j=0}^{6} \vec{u}_{j} \varepsilon^{j}+\mathcal{O}\left(\varepsilon^{7}\right)$, where each coefficient $\vec{u}_{j}$ has uniform transcendental weight $j$.

The UT integral basis construction is a complicated task even with special tools designed to attack the problem $[43,44]$. Fortunately, with already available results we can easily avoid all these difficulties.

First, we notice that if the integrals with arbitrary $z$ have UT weight, this property also holds for the integrals at the symmetric point $\left(z \rightarrow e^{\frac{i \pi}{3}}\right)$. The weight of GPLs and all $z$-dependent prefactors do not change transcendentality after taking the limit, and we can focus on constructing UT basis for auxiliary integrals.

Second, due to the UT property, the DE system for UT integrals is also in $\varepsilon$-form. Our goal is to find a transformation matrix between canonical master integrals from section 3 and the new UT basis. As mentioned in section 3, the last step of reduction to the $\varepsilon$-form has a freedom, and we are looking for the transformation to UT basis by varying these parameters.

Another important observation is that for the $z$-dependent integrals with UT weight, this property also holds when considering their expansion near singular points. For example leading terms of expansion in $z \rightarrow 1$ used for fixing boundary conditions in section 4 .

$$
J_{\mathrm{UT}}(\bar{z}) \sim \sum_{i} \bar{z}^{a_{i} \varepsilon} C_{i}
$$

Since $\varepsilon$-expansion of the prefactor $\bar{z}^{a_{i} \varepsilon}$ in (5.1) has transcendental weight zero, all coefficients $C_{i}$ for expansion of the UT integral $J_{\mathrm{UT}}$ also have UT weight. In considered limit, all coefficients $C_{i}$ are built from massless propagator integrals only. After reduction to master integrals, most of them are known for arbitrary $D$, and for the remaining, transformation to the UT basis is known from [45]. In this way, we find transformation matrix $T_{\mathrm{UT}}$ by analyzing expansion coefficients in the limit $z \rightarrow 1$.

All UT integrals constructed in this way are regular in the limit $z \rightarrow e^{\frac{i \pi}{3}}$ and going back to the original integrals and then reducing to the basis of symmetric point integrals, we obtain representation for integrals of our interest in terms of pure functions. 


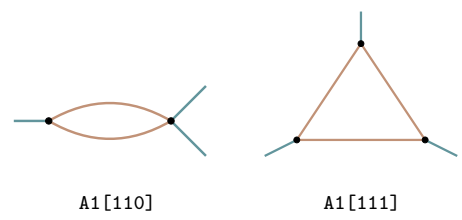

Table 4. One-loop master integrals.

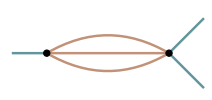

A2 [0110100]

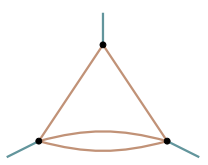

A2 [0110110]

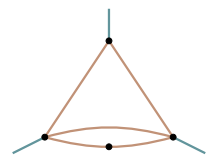

A2 [0210110]

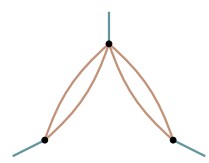

A2 [1101010]

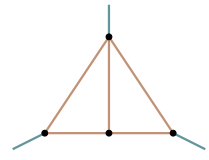

A2 [1101110]

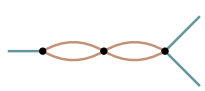

A2 [1111000]
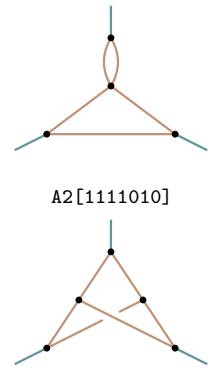

B2 [1111110]

Table 5. Two-loop master integrals.

\section{Results and conclusion}

In addition to the described method, we use IBP reduction to the new basis of finite integrals to follow the strategy described in the paper [26]. With package HyperInt [46], we find an analytical solution for several integrals, except the most complicated, which is in complete agreement with results of the previous sections. All integrals calculated in the paper were checked numerically with the sector decomposition approach implemented in the package pySecDec [47].

In tables 4, 5 and 6, we present all master integrals needed for calculations at the symmetric point up to the three-loop order. Analytical results for integrals can be found in supplementary files to the paper and contain a transformation matrix to the basis of UT weight functions and expansion of latter up to the transcendental weight six.

Up to the three-loop level, expansion of the basis functions is expressed in terms of HPLs with the sixth-root of unity argument. Instead of the basis constructed in paper [48], which has an attractive feature, that reduction rules for real(imaginary) part of HPLs contain only real(imaginary) parts of basis functions. We construct a new basis allowing us to present results in a more compact form. Also, our basis contains as basis elements products of lower weight functions, which simplifies products of lower-loop integrals during renormalization. The total number of elements $N_{w}$ at weight $w$ is the same $N_{w}=2 F_{2 w}$, where $F_{n}$ is the $n$-th Fibonacci number and explicitly is given by $N_{w}=\{2,6,16,42,110,288\}$ for the weights considered in the paper.

In the paper, we calculated all massless three-point master integrals at the symmetric point. As the solution method, we apply differential equations for specially constructed auxiliary integrals and fix boundary conditions from the large momentum expansion. Results for integrals are expressed through the basis of functions with uniform transcendental weight. We provide expansion in $\varepsilon$ for these functions in terms of the HPLs with the sixth 


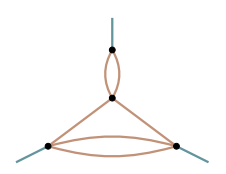

A3 [001010111100]

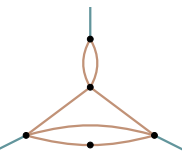

A3 [002010111100]

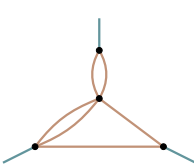

A3 [011010111000]

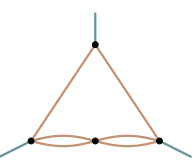

A3 [011110110000]

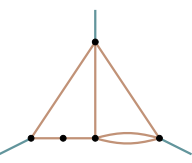

A3 [021110011000]

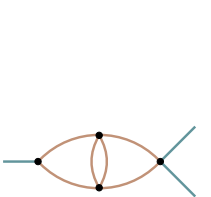

A3[110011101000]

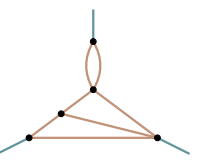

A3[111100011010]

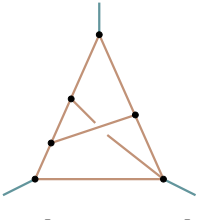

B3 [011111100110]

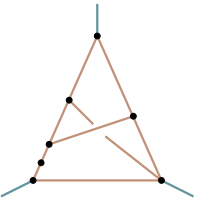

В3 [021111100110]

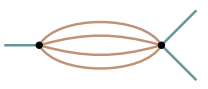

A3 [001101100000]

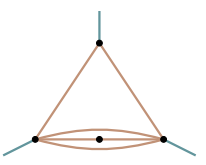

A3 [002101110000]

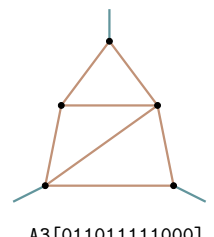

A3 [011011111000]

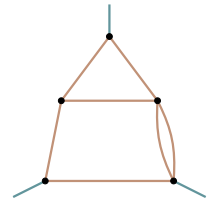

А3 [011110111000]

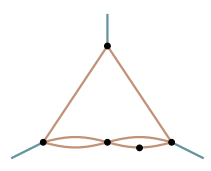

A3 [021110110000]

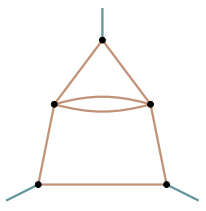

A3[110011111000]

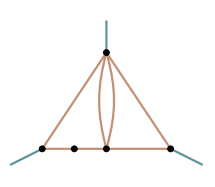

A3 [210011011000]

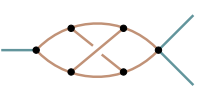

B3 [011111101010]

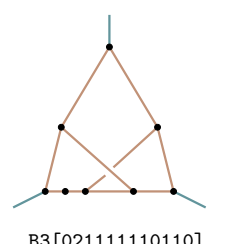

B3 [021111110110]

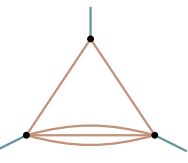

A3 [001101110000]

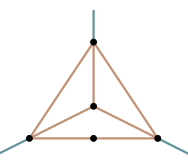

A3 [002111011000]

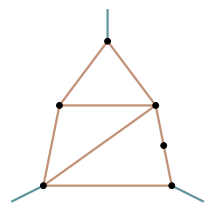

A3 [011011211000]

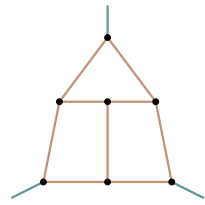

A3 [011111110110]

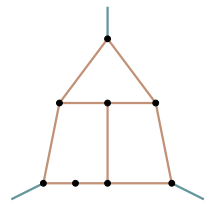

A3 [021111110110]

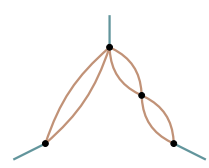

A3 [111000011010]

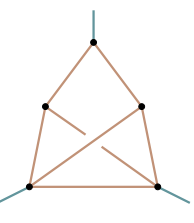

B3 [001111100110]

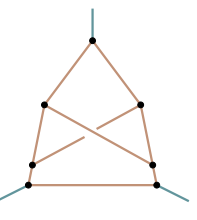

B3 [011111101110]

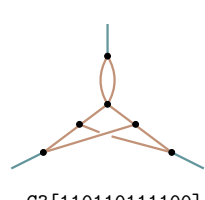

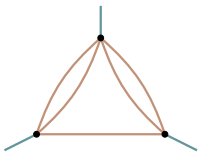

A3 [001110011000]

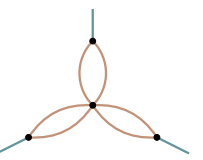

A3 [011000110110]
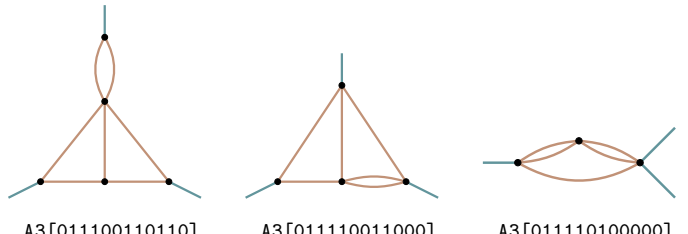

A3 [011110100000]

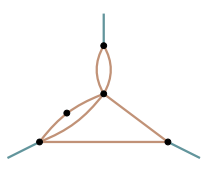

A3 [012010111000]

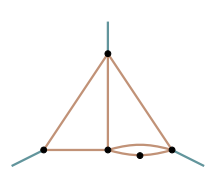

A3 [012110011000]

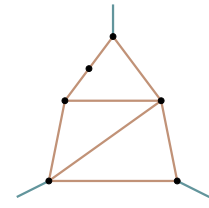

A3 [021011111000]

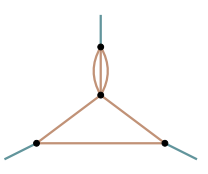

A3 [101001111000]

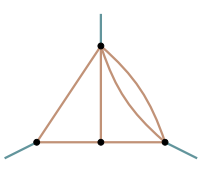

A3 [101011011000]

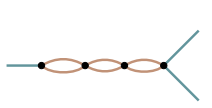

A3 [111000101010]

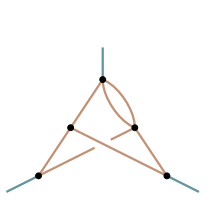

B3 [010101110110]

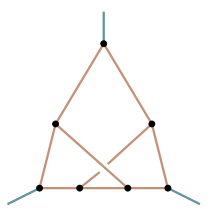

B3 [011111110110]

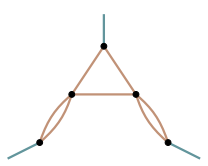

A3 [111000110110]

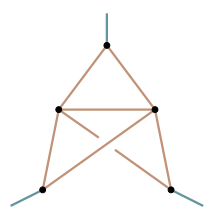

B3 [011001110110]

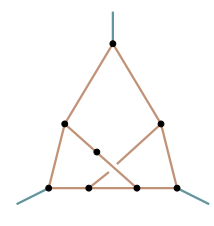

B3 [011211110110]
A3 [110011011000]
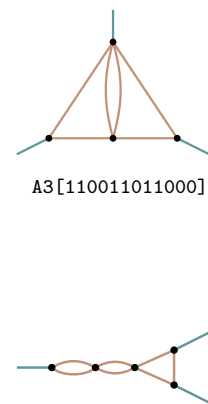

A3 [111000111010]

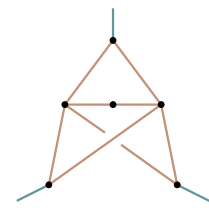

B3 [011002110110]

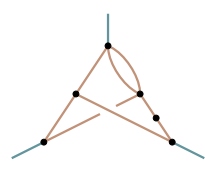

Table 6. Three-loop master integrals. 
root of unity argument up to the transcendental weight six. The obtained result can find its application in future calculations in the RI/SMOM scheme and as the boundary conditions for three-point integrals in more general kinematics.

\section{Acknowledgments}

We gratefully acknowledge Alexander Bednyakov for collaboration on [12, 13]. We thank Roman Lee for his valuable suggestions and discussions. We are grateful to the Joint Institute for Nuclear Research for letting us use their supercomputer "Govorun". This work was supported by Russian Science Foundation, grant 20-12-00205.

Open Access. This article is distributed under the terms of the Creative Commons Attribution License (CC-BY 4.0), which permits any use, distribution and reproduction in any medium, provided the original author(s) and source are credited.

\section{References}

[1] P.A. Baikov, K.G. Chetyrkin and J.H. Kühn, Five-Loop Running of the QCD coupling constant, Phys. Rev. Lett. 118 (2017) 082002 [arXiv:1606.08659] [INSPIRE].

[2] T. Luthe, A. Maier, P. Marquard and Y. Schröder, The five-loop $\beta$-function for a general gauge group and anomalous dimensions beyond Feynman gauge, JHEP 10 (2017) 166 [arXiv: 1709.07718] [INSPIRE].

[3] F. Herzog, B. Ruijl, T. Ueda, J.A.M. Vermaseren and A. Vogt, The five-loop $\beta$-function of Yang-Mills theory with fermions, JHEP 02 (2017) 090 [arXiv: 1701.01404] [INSPIRE].

[4] K.G. Chetyrkin, G. Falcioni, F. Herzog and J.A.M. Vermaseren, Five-loop renormalisation of QCD in covariant gauges, JHEP 10 (2017) 179 [Addendum ibid. 12 (2017) 006] [arXiv: 1709.08541] [INSPIRE].

[5] G. 't Hooft, Dimensional regularization and the renormalization group, Nucl. Phys. B 61 (1973) 455 [INSPIRE].

[6] W. Celmaster and R.J. Gonsalves, The Renormalization Prescription Dependence of the QCD Coupling Constant, Phys. Rev. D 20 (1979) 1420 [InSPIRE].

[7] J.A. Gracey, Three loop QCD MOM $\beta$-functions, Phys. Lett. B 700 (2011) 79 [arXiv: 1104.5382] [INSPIRE].

[8] L.G. Almeida and C. Sturm, Two-loop matching factors for light quark masses and three-loop mass anomalous dimensions in the RI/SMOM schemes, Phys. Rev. D 82 (2010) 054017 [arXiv: 1004.4613] [INSPIRE].

[9] J.A. Gracey, RI'/SMOM scheme amplitudes for quark currents at two loops, Eur. Phys. J. C 71 (2011) 1567 [arXiv:1101.5266] [inSPIRE].

[10] J.A. Gracey, Two loop renormalization of the $N=2$ Wilson operator in the RI'/SMOM scheme, JHEP 03 (2011) 109 [arXiv:1103.2055] [INSPIRE].

[11] J.A. Gracey, Amplitudes for the $N=3$ moment of the Wilson operator at two loops in the RI/'SMOM scheme, Phys. Rev. D 84 (2011) 016002 [arXiv:1105.2138] [InSPIRE].

[12] A. Bednyakov and A. Pikelner, Quark masses: N3LO bridge from RI/SMOM to $\overline{\mathrm{MS}}$ scheme, Phys. Rev. D 101 (2020) 091501 [arXiv:2002.12758] [INSPIRE]. 
[13] A. Bednyakov and A. Pikelner, Four-loop QCD MOM $\beta$-functions from the three-loop vertices at the symmetric point, Phys. Rev. D 101 (2020) 071502 [arXiv: 2002.02875] [INSPIRE].

[14] B.A. Kniehl and O.L. Veretin, Moments $N=2$ and $N=3$ of the Wilson twist-two operators at three loops in the RI'/SMOM scheme, Nucl. Phys. B 961 (2020) 115229 [arXiv:2009.11325] [INSPIRE].

[15] B.A. Kniehl and O.L. Veretin, Bilinear quark operators in the RI/SMOM scheme at three loops, Phys. Lett. B 804 (2020) 135398 [arXiv:2002.10894] [InSPIRE].

[16] K.G. Chetyrkin and T. Seidensticker, Two loop QCD vertices and three loop MOM $\beta$-functions, Phys. Lett. B 495 (2000) 74 [hep-ph/0008094] [INSPIRE].

[17] F.V. Tkachov, A Theorem on Analytical Calculability of Four Loop Renormalization Group Functions, Phys. Lett. B 100 (1981) 65 [InSPIRE].

[18] K.G. Chetyrkin and F.V. Tkachov, Integration by Parts: The Algorithm to Calculate $\beta$-functions in 4 Loops, Nucl. Phys. B 192 (1981) 159 [InSPIRE].

[19] A.I. Davydychev, Recursive algorithm of evaluating vertex type Feynman integrals, J. Phys. A 25 (1992) 5587 [INSPIRE].

[20] N.I. Usyukina and A.I. Davydychev, New results for two loop off-shell three point diagrams, Phys. Lett. B 332 (1994) 159 [hep-ph/9402223] [INSPIRE].

[21] T.G. Birthwright, E.W.N. Glover and P. Marquard, Master integrals for massless two-loop vertex diagrams with three offshell legs, JHEP 09 (2004) 042 [hep-ph/0407343] [INSPIRE].

[22] N.I. Usyukina and A.I. Davydychev, Exact results for three and four point ladder diagrams with an arbitrary number of rungs, Phys. Lett. B 305 (1993) 136 [InSPIRE].

[23] F. Chavez and C. Duhr, Three-mass triangle integrals and single-valued polylogarithms, JHEP 11 (2012) 114 [arXiv: 1209.2722] [INSPIRE].

[24] E. Panzer, Feynman integrals and hyperlogarithms, Ph.D. Thesis, Humboldt University, Berlin Germany (2015) [arXiv: 1506.07243] [INSPIRE].

[25] J.M. Henn, Multiloop integrals in dimensional regularization made simple, Phys. Rev. Lett. 110 (2013) 251601 [arXiv:1304.1806] [INSPIRE].

[26] A. von Manteuffel, E. Panzer and R.M. Schabinger, A quasi-finite basis for multi-loop Feynman integrals, JHEP 02 (2015) 120 [arXiv: 1411.7392] [inSPIRE].

[27] S. Laporta, High precision calculation of multiloop Feynman integrals by difference equations, Int. J. Mod. Phys. A 15 (2000) 5087 [hep-ph/0102033] [INSPIRE].

[28] A. von Manteuffel and C. Studerus, Reduze 2 - Distributed Feynman Integral Reduction, arXiv: 1201.4330 [INSPIRE].

[29] S.G. Gorishnii, S.A. Larin, L.R. Surguladze and F.V. Tkachov, Mincer: Program for Multiloop Calculations in Quantum Field Theory for the Schoonschip System, Comput. Phys. Commun. 55 (1989) 381 [INSPIRE].

[30] S.A. Larin, F.V. Tkachov and J.A.M. Vermaseren, The FORM version of MINCER, NIKHEF-H-91-18 (1991).

[31] T. Gehrmann, E.W.N. Glover, T. Huber, N. Ikizlerli and C. Studerus, Calculation of the quark and gluon form factors to three loops in QCD, JHEP 06 (2010) 094 [arXiv: 1004.3653] [INSPIRE]. 
[32] R.N. Lee, A.V. Smirnov and V.A. Smirnov, Analytic Results for Massless Three-Loop Form Factors, JHEP 04 (2010) 020 [arXiv: 1001.2887] [InSPIRE].

[33] A. von Manteuffel, E. Panzer and R.M. Schabinger, On the Computation of Form Factors in Massless QCD with Finite Master Integrals, Phys. Rev. D 93 (2016) 125014 [arXiv: 1510.06758] [INSPIRE].

[34] R.N. Lee, Reducing differential equations for multiloop master integrals, JHEP 04 (2015) 108 [arXiv: 1411.0911] [INSPIRE].

[35] M. Prausa, epsilon: A tool to find a canonical basis of master integrals, Comput. Phys. Commun. 219 (2017) 361 [arXiv: 1701.00725] [INSPIRE].

[36] A.B. Goncharov, Multiple polylogarithms and mixed Tate motives, math/0103059 [INSPIRE].

[37] J.M. Henn, A.V. Smirnov and V.A. Smirnov, Evaluating Multiple Polylogarithm Values at Sixth Roots of Unity up to Weight Six, Nucl. Phys. B 919 (2017) 315 [arXiv:1512.08389] [INSPIRE].

[38] F. Dulat and B. Mistlberger, Real-Virtual-Virtual contributions to the inclusive Higgs cross section at N3LO, arXiv:1411.3586 [INSPIRE].

[39] R.N. Lee, A.V. Smirnov and V.A. Smirnov, Solving differential equations for Feynman integrals by expansions near singular points, JHEP 03 (2018) 008 [arXiv:1709.07525] [INSPIRE].

[40] R. Harlander, T. Seidensticker and M. Steinhauser, Complete corrections of $O\left(\alpha \alpha_{s}\right)$ to the decay of the $Z$ boson into bottom quarks, Phys. Lett. B 426 (1998) 125 [hep-ph/9712228] [INSPIRE].

[41] T. Seidensticker, Automatic application of successive asymptotic expansions of Feynman diagrams, in 6th International Workshop on New Computing Techniques in Physics Research: Software Engineering, Artificial Intelligence Neural Nets, Genetic Algorithms, Symbolic Algebra, Automatic Calculation, Heraklion Greece (1999) [hep-ph/9905298] [INSPIRE].

[42] R. Harlander, Asymptotic expansions: Methods and applications, Acta Phys. Polon. B 30 (1999) 3443 [hep-ph/9910496] [INSPIRE].

[43] C. Dlapa, J. Henn and K. Yan, Deriving canonical differential equations for Feynman integrals from a single uniform weight integral, JHEP 05 (2020) 025 [arXiv:2002.02340] [INSPIRE].

[44] J. Henn, B. Mistlberger, V.A. Smirnov and P. Wasser, Constructing d-log integrands and computing master integrals for three-loop four-particle scattering, JHEP 04 (2020) 167 [arXiv: 2002.09492] [INSPIRE].

[45] R.N. Lee, A.V. Smirnov and V.A. Smirnov, Master Integrals for Four-Loop Massless Propagators up to Transcendentality Weight Twelve, Nucl. Phys. B 856 (2012) 95 [arXiv: 1108.0732] [INSPIRE].

[46] E. Panzer, Algorithms for the symbolic integration of hyperlogarithms with applications to Feynman integrals, Comput. Phys. Commun. 188 (2015) 148 [arXiv:1403.3385] [INSPIRE].

[47] S. Borowka et al., pySecDec: a toolbox for the numerical evaluation of multi-scale integrals, Comput. Phys. Commun. 222 (2018) 313 [arXiv:1703.09692] [INSPIRE].

[48] B.A. Kniehl, A.F. Pikelner and O.L. Veretin, Three-loop massive tadpoles and polylogarithms through weight six, JHEP 08 (2017) 024 [arXiv:1705.05136] [INSPIRE]. 